\title{
Theory of ac electrokinetic behavior of spheroidal cell suspensions with an intrinsic dispersion
}

\author{
Lei $\mathrm{Gao}^{1,2}$, J. P. Huang ${ }^{1}$ and K. W. Yu ${ }^{1}$ \\ ${ }^{1}$ Department of Physics, The Chinese University of Hong Kong, Shatin, N.T., Hong Kong, China \\ 2 Department of Physics, Suzhou University, Suzhou 215006, China
}

\begin{abstract}
The dielectric dispersion, dielectrophoretic (DEP) and electrorotational (ER) spectra of spheroidal biological cell suspensions with an intrinsic dispersion in the constituent dielectric constants are investigated. By means of the spectral representation method, we express analytically the characteristic frequencies and dispersion strengths both for the effective dielectric constant and the Clausius-Mossotti factor (CMF). We identify four and six characteristic frequencies for the effective dielectric spectra and CMF respectively, all of them being dependent on the depolarization factor (or the cell shape). The analytical results allow us to examine the effects of the cell shape, the dispersion strength and the intrinsic frequency on the dielectric dispersion, DEP and ER spectra. Furthermore, we include the local-field effects due to the mutual interactions between cells in a dense suspension, and study the dependence of co-field or anti-field dispersion peaks on the volume fractions.
\end{abstract}

PACS Number(s): 87.18.-h, 82.70.-y, 77.22.Gm, 77.84.Nh 


\section{INTRODUCTION}

The polarization of biological cells has a wide range of practical applications like manipulation, trapping or separation of biological cells [1,2]. Dielectric spectroscopy [3], dielectrophoresis (DEP) [4] and electrorotation (ER) [5] offer a unique capability of monitoring the dielectric properties of dispersions of colloids and biological cells. Under the action of external fields, these particles exhibit rich fluid-dynamic behaviors as well as various dielectric responses. Hence, it is of importance to investigate their frequency-dependent responses to ac electric fields, which yields valuable information on the structural (MaxwellWagner) polarization effects. The polarization is characterized by a variety of characteristic frequency-dependent changes known as the dielectric dispersion, whose spectra are helpful to analyze the inhomogeneous systems, including biological cell suspensions and tissues [6]. Many factors exert influences on the effective dielectric behavior of the system such as orientation of dipoles, surface conductance and the cell shape [7]. However, some factors can be dominant at certain ranges of frequencies (for example, the experimental data revealed that the low frequency subdispersions were dependent on the cell shape [8]).

The DEP is used to describe the motion of the particles caused by the dielectric polarization effects in non-uniform electric field [9]. The DEP force drives the particles towards high intensity (positive DEP) or towards regions of minimal field intensity (negative DEP). Because of the interaction (i.e., DEP force) between the induced dipole and the external electric field, the particles can be levitated in the medium [10].

The ER behavior is caused by the existence of a phase difference between the field-induced dipole moment and the external rotating field, which results in a torque to cause the particle to rotate. In the dilute limit, the ER of individual cell can be predicted by ignoring the mutual interaction between the cells, and hence may be considered as an isolated particle in rotation [11].

In this paper, we will apply the spectral representation theory [12] to investigate the ac electrokinetic behavior including the dielectric dispersion, DEP and ER spectra of biological cell suspensions. The object of the present investigation is threefold. First, it is instructive to consider the effect of the intrinsic dielectric dispersion. Actually, such an intrinsic dielectric dispersion often occurs due to the surface conductivity $[13,14]$ or the inhomogeneous structure such as the coated shells of biological cells or colloidal suspensions [15]. Secondly, many cells exist in the form of the nonspherical shape such as the fission yeast cell [8] and the red blood cell [16], and hence the effect of cell shape needs to be considered $[17,18]$. Thirdly, the volume fractions of the biological cell suspensions are generally not in the dilute limit and can even exceed $0.1[6,8,19]$, thus the local field effect due to the average electrostatic interaction between cells must be taken into account [20]. In view of this, the effective dielectric dispersion spectra will be studied based on Maxwell-Garnett type approximation [21], which involves an exact calculation of the field induced in the host medium by a single ellipsoidal cell and an approximate treatment of its distortion by the electrostatic interaction between different cell suspensions. Moreover, the Clausius-Mossotti factor (CMF), which determines the DEP and ER spectra, will be modified by replacing the dielectric constant of the host medium with the effective one.

Being beyond this work, we noticed an alternative paper [22], in which the authors considered the electrorotation and levitation of spherical cells or colloidal particles with/without 
the membrane-covered shells in the dilute limit. However, our investigation can be valid not only for nonspherical cell suspensions without membrane-covered shells, but also for nondilute limit suspensions. Furthermore, we adopt the spectral representation method [12], which offers the advantage of the separation of material parameters from the geometric information, to simplify the derivation of the analytical expressions for the characteristic frequencies and dispersion strengths of the effective dielectric permittivity and the CMF, respectively.

\section{FORMALISM}

We consider a composite system in which biological cells of dielectric constant $\tilde{\epsilon}_{1}$ with the volume fraction $p$ are dispersed in an isotropic host medium with dilectric constant $\tilde{\epsilon}_{2}=\epsilon_{2}+\sigma_{2} /(j 2 \pi f)$ with $j=\sqrt{-1}, f$ being the frequency of the applied field. $\tilde{\epsilon}_{1}$ is assumed to exhibit an intrinsic dielectric dispersion, i.e.,

$$
\tilde{\epsilon}_{1}=\epsilon_{1}+\frac{\triangle \epsilon_{1}}{1+j f / f_{1}}+\frac{\sigma_{1}}{j 2 \pi f},
$$

where $\epsilon_{1}\left(\sigma_{1}\right)$ is the limiting high-frequency (low-frequency) dielectric constant (conductivity), $\triangle \epsilon_{1}$ represents the dielectric dispersion strength with a characteristic frequency $f_{1}$. Biological cells are further assumed to be spheroidal in shape. Such an assumption is supported by Bohren et al [23] who suggested that dielectric dispersion spectra of particles of arbitrary shape can be approximated by these of spheroidal particles.

Then we will adopt the spectral representation to investigate the ac electrokinetic behavior such as the dielectric dispersion, dielectrophoretic and electrorotational spectra of an inhomogeneous system in which spheroidal biological cells are randomly distributed.

\section{A. Dielectric dispersion spectrum}

Generally, for cell suspensions of arbitrary shape, the spectral representation can only be solved numerically. However, for randomly-oriented spheroidal cell suspensions, within the mean-field theory, the effective complex dielectric constant $\tilde{\epsilon}_{e}$ can be written as [21]

$$
\tilde{\epsilon}_{e}=\tilde{\epsilon}_{2} \frac{1+p\left[b_{z}\left(1-L_{z}\right)+2 b_{x y}\left(1-L_{x y}\right)\right]}{1-p\left(b_{z} L_{z}+2 b_{x y} L_{x y}\right)},
$$

where $b_{k} \equiv\left(\tilde{\epsilon}_{1}-\tilde{\epsilon}_{2}\right) /\left\{3\left[\tilde{\epsilon}_{2}+L_{k}\left(\tilde{\epsilon}_{1}-\tilde{\epsilon}_{2}\right)\right]\right\} \quad(k=z, x y)$ is called the Clausius-Mossotti factor $(\mathrm{CMF})$, and $L_{z}\left[L_{x y}=\left(1-L_{z}\right) / 2\right]$ are the depolarization factors along the $z(x$ or $y)$ axis of the spheroids. These depolarization factors depend on the aspect ratio $q \equiv c / a[21]$, where $a$ $(=b), c$ are the semi-axes of a spheroid along the Cartesian axes. For the prolate-spheroidal cell $(q>1)$, we have $0<L_{z}<1 / 3$; while for the oblate-spheroidal one $(q<1)$, we have $1 / 3<L_{z}<1$. Actually, once a $q$ is given, $L_{z}$ can be obtained uniquely and thus used to indicate the shape of spheroidal particles.

By invoking the spectral representation and introducing the dimensionless parameter $\tilde{s} \equiv \tilde{\epsilon}_{2} /\left(\tilde{\epsilon}_{2}-\tilde{\epsilon}_{1}\right)$, we rewrite Eq.(2) as 


$$
\tilde{\epsilon}_{e}=\tilde{\epsilon}_{2}\left(1-\frac{W_{1}}{\tilde{s}-x_{1}}-\frac{W_{2}}{\tilde{s}-x_{2}}\right)
$$

where the poles $x_{1}$ and $x_{2}$ are given as

$$
x_{1,2}=\frac{1}{12}\left[3-2 p+3 L_{z} \pm \sqrt{\left(3-2 p+3 L_{z}\right)^{2}-72(1-p) L_{z}\left(1-L_{z}\right)}\right],
$$

Correspondingly, the residues $W_{1}$ and $W_{2}$ have

$$
W_{1}=\frac{p\left(1+3 L_{z}-6 x_{1}\right)}{6\left(x_{2}-x_{1}\right)}, W_{2}=\frac{p\left(1+3 L_{z}-6 x_{2}\right)}{6\left(x_{1}-x_{2}\right)} .
$$

It is easy to check that $W_{1}$ and $W_{2}$ satisfy the sum rule $W_{1}+W_{2}=p$.

In order to obtain the analytic expressions for the dielectric dispersion strengths and the characteristic frequencies of the dielectric dispersion spectra, we then introduce two contrast parameters $s=\epsilon_{2} /\left(\epsilon_{2}-\epsilon_{1}\right)$ and $t=\sigma_{2} /\left(\sigma_{2}-\sigma_{1}\right)$, firstly defined by Lei et al [7], and derive an equality for biological cells possessing an intrinsic dispersion,

$$
\frac{1}{\tilde{s}-x}=\frac{1}{s-x}+\frac{A(x)}{1+j f / f_{c 1}(x)}+\frac{B(x)}{1+j f / f_{c 2}(x)},
$$

where

$$
f_{c 1, c 2}(x)=\frac{f_{1} f_{2}+f_{2} f_{3}+f_{3} f_{1} \mp \sqrt{\left(f_{1} f_{2}+f_{2} f_{3}+f_{3} f_{1}\right)^{2}-4 f_{1} f_{2} f_{3}^{2}}}{2 f_{3}}
$$

and

$$
\begin{aligned}
& A(x)=\left[\frac{s-t}{(s-x)(t-x)} \cdot\left(\frac{f_{1}-f_{c 1}}{f_{1} f_{c 1}}\right)+\frac{s}{(s-x) x} \cdot \frac{1}{f_{3}}\right] \frac{f_{1} f_{2}}{f_{c 2}(x)-f_{c 1}(x)}, \\
& B(x)=\left[\frac{s-t}{(s-x)(t-x)} \cdot\left(\frac{f_{c 2}-f_{1}}{f_{1} f_{c 2}}\right)-\frac{s}{(s-x) x} \cdot \frac{1}{f_{3}}\right] \frac{f_{1} f_{2}}{f_{c 2}(x)-f_{c 1}(x)},
\end{aligned}
$$

with

$$
f_{2}(x)=\frac{1}{2 \pi} \cdot \frac{s \sigma_{2}(t-x)}{\epsilon_{2} t(s-x)}, f_{3}(x)=\frac{1}{2 \pi} \cdot \frac{\sigma_{2}(t-x)}{x \triangle \epsilon_{1} t} .
$$

After simple manipulations, we can express Eq.(3) as,

$$
\tilde{\epsilon_{e}}=\epsilon_{H}+\sum_{i=1}^{4} \frac{\triangle \epsilon_{e i}}{1+j\left(f / f_{e i}\right)}+\frac{\sigma_{L}}{j 2 \pi f} \equiv \epsilon_{e}+\frac{\sigma_{e}}{j 2 \pi f}
$$

with

$$
\epsilon_{H}=\epsilon_{2}\left(1-\frac{W_{1}}{s-x_{1}}-\frac{W_{2}}{s-x_{2}}\right), \quad \sigma_{L}=\sigma_{2}\left(1-\frac{W_{1}}{t-x_{1}}-\frac{W_{2}}{t-x_{2}}\right)
$$

and 


$$
\begin{aligned}
& \triangle \epsilon_{e 1}=W_{1} A\left(x_{1}\right)\left(\frac{\sigma_{2}}{2 \pi f_{c 1}\left(x_{1}\right)}-\epsilon_{2}\right), f_{e 1}=f_{c 1}\left(x_{1}\right) \\
& \triangle \epsilon_{e 2}=W_{1} B\left(x_{1}\right)\left(\frac{\sigma_{2}}{2 \pi f_{c 2}\left(x_{1}\right)}-\epsilon_{2}\right), f_{e 2}=f_{c 2}\left(x_{1}\right)
\end{aligned}
$$

Similarly, $\triangle \epsilon_{e i}$ and $f_{e i}$ for $i=3,4$ can be easily got by our replacing $W_{1}$ and $x_{1}$ with $W_{2}$ and $x_{2}$ from above two equations.

Thus, within the spectral representation, for spheroidal suspensions with one intrinsic dispersion, we successfully obtain four characteristic freqencies $f_{e i}(i=1,4)$ with the dispersion strengths $\triangle \epsilon_{e i}$ in terms of the geometric parameters $\left(L_{z}\right.$ and $\left.p\right)$ as well as physical parameters $\left(s, t, \triangle \epsilon_{1}, \sigma_{2}\right.$ and $\left.\epsilon_{2}\right)$.

In Fig.1, we numerically calculate four characteristic frequencies and dispersion strengths against $L_{z}$. As is evident from the figure, the characteristic freqencies and the corresponding dispersion strengths are strongly dependent on the shape of cell suspensions and exhibit nonmonotonical behavior with increasing $L_{z}$. For these four characteristic frequencies, two of them are located at frequencies higher than $10^{6} \mathrm{~Hz}$, while another two are at frequencies lower than $10^{6} \mathrm{~Hz}$. Such a separation results from the fact that we take into account one intrinsic dispersion in the dielectric cell suspensions. Furthermore, due to the nonspherical shape of cell suspensions, there are two characteristic frequencies in the high-frequency (or low-frequency) region. When the shape is far from spherical (say $L_{z} \rightarrow 0$ or 1 ), the difference between these two frequencies becomes large. Conversely, for spherical inclusions $\left(L_{z}=1 / 3\right)$, only two dispersion strengths are nonzero, leading to two-main steps in the dielectric dispersion spectra, as will be shown in the next section.

\section{B. dielectrophoretic and electrorotational spectra}

The spectral form of Eq.(6) also allows us to investigate the dielectrophoretic and electrorotational behavior.

It is known that the time average DEP force $F(f)$ and ER torque $\Gamma(f)$ excerted on the spheroidal cell suspensions with $z$-orientation parallel to the electric field are expressed as $[24,25]$

$$
F(f)=1.5 v \tilde{\epsilon}_{2} \operatorname{Re}\left[b_{z}\left(\tilde{\epsilon}_{1}, \tilde{\epsilon}_{2}\right)\right]\left|\nabla \mathbf{E}_{(r m s)}^{2}\right|, \Gamma(f)=-3 v \tilde{\epsilon}_{2} \operatorname{Im}\left[b_{z}\left(\tilde{\epsilon}_{1}, \tilde{\epsilon}_{2}\right)\right] \mathbf{E}_{0}^{2},
$$

where $v$ is the volume of spheroidal cell, $\mathbf{E}_{(r m s)}$ is the root-mean square magnitude of the imposed ac electric field and $\operatorname{Re}[\cdots], \operatorname{Im}[\cdots]$ represent the real and imaginary parts of $b_{z}$, which can be written under the spectral representation as

$$
b_{z}\left(\tilde{\epsilon}_{1}, \tilde{\epsilon}_{2}\right)=\frac{1}{3} \cdot \frac{\tilde{\epsilon}_{1}-\tilde{\epsilon}_{2}}{\tilde{\epsilon}_{2}+L_{z}\left(\tilde{\epsilon}_{1}-\tilde{\epsilon}_{2}\right)}=-\frac{1}{3} \cdot \frac{1}{\tilde{s}-L_{z}}
$$

Eq.(16) is independent of the volume fraction $p$ and thus is valid only in the dilute limit [22]. However, for non-dilute volume fractions, we must consider the local field effect due to mutual interaction between the spheroidal cells and modify Eq.(16) as,

$$
b_{z}\left(\tilde{\epsilon}_{1}, \tilde{\epsilon}_{2}\right)=\frac{1}{3} \cdot \frac{\tilde{\epsilon}_{1}-\tilde{\epsilon}_{e}}{\tilde{\epsilon}_{e}+L_{z}\left(\tilde{\epsilon}_{1}-\tilde{\epsilon}_{e}\right)} .
$$


Substituting Eq.(2) into (17), we have

$$
\begin{aligned}
b_{z} & =\frac{2(p-1) \tilde{s}^{2}+(1-p)\left(1+L_{z}\right) \tilde{s}-(1-p) L_{z}\left(1-L_{z}\right)}{6 \tilde{s}^{3}-\left(3+4 p+9 L_{z}-6 p L_{z}\right) \tilde{s}^{2}+\left(p+6 L_{z}-3 p L_{z}\right) \tilde{s}+3(p-1) L_{z}^{2}\left(1-L_{z}\right)} \\
& =\frac{V_{1}}{\tilde{s}-s_{1}}+\frac{V_{2}}{\tilde{s}-s_{2}}+\frac{V_{3}}{\tilde{s}-s_{3}},
\end{aligned}
$$

where $s_{i}(i=1,2,3)$ are three roots of polynomial equations

$$
y^{3}-\frac{3+4 p+9 L_{z}-6 p L_{z}}{6} y^{2}+\frac{p+6 L_{z}-3 p L_{z}}{6} y+\frac{(p-1) L_{z}^{2}\left(1-L_{z}\right)}{2}=0,
$$

while the residues $V_{1}, V_{2}$ and $V_{3}$ can be obtained from

$$
\begin{aligned}
V_{1}+V_{2}+V_{3} & =\frac{1}{3}(p-1) \\
V_{1}\left(s_{2}+s_{3}\right)+V_{2}\left(s_{3}+s_{1}\right)+V_{3}\left(s_{1}+s_{2}\right) & =\frac{1}{6}(p-1)\left(1+L_{z}\right) \\
V_{1} s_{2} s_{3}+V_{2} s_{1} s_{3}+V_{3} s_{1} s_{2} & =\frac{1}{6}(p-1) L_{z}\left(1-L_{z}\right) .
\end{aligned}
$$

Note that these residues again satisfy the sum rule $V_{1}+V_{2}+V_{3}=-(1-p) / 3$.

Introducing Eq.(6) into Eq.(18) leads to

$$
b_{z}=\sum_{i=1}^{3}\left[\frac{V_{i}}{s-s_{i}}+\frac{V_{i} A\left(s_{i}\right)}{1+j f / f_{c 1}\left(s_{i}\right)}+\frac{V_{i} B\left(s_{i}\right)}{1+j f / f_{c 2}\left(s_{i}\right)}\right] .
$$

The normalized DEP force and normalized ER torque can be defined as

$$
N D E P=\operatorname{Re}\left[b_{z}\left(\tilde{\epsilon}_{1}, \tilde{\epsilon}_{2}\right)\right]=\sum_{i=1}^{3}\left[\frac{V_{i}}{s-s_{i}}+\frac{V_{i} A\left(s_{i}\right)}{1+\left[f / f_{c 1}\left(s_{i}\right)\right]^{2}}+\frac{V_{i} B\left(s_{i}\right)}{1+\left[f / f_{c 2}\left(s_{i}\right)\right]^{2}}\right],
$$

and

$$
N E R=-\operatorname{Im}\left[b_{z}\left(\tilde{\epsilon}_{1}, \tilde{\epsilon}_{2}\right)\right]=\sum_{i=1}^{3}\left[\frac{V_{i} A\left(s_{i}\right) f / f_{c 1}\left(s_{i}\right)}{1+\left[f / f_{c 1}\left(s_{i}\right)\right]^{2}}+\frac{V_{i} B\left(s_{i}\right) f / f_{c 2}\left(s_{i}\right)}{1+\left[f / f_{c 2}\left(s_{i}\right)\right]^{2}}\right] .
$$

Based upon the spectral representation, the expressions for the dispersion strengths and characteristic frequencies of CMF are also explicitly obtained. Six CMF characteristic frequencies and dispersion strengths against $L_{z}$ are shown in Fig.2.

Again, $L_{z}$ plays an important role in determining the CMF characteristic frequencies and the corresponding strengths. Within our model, six CMF characteristic frequencies are predicted; three of them are located below $10^{6} \mathrm{~Hz}$ and another three do above $10^{6} \mathrm{~Hz}$, similar as that observed in Fig.1. Due to this, the DEP (or ER) spectra are maining characterized by two-step rapid changes (or two dominant peaks). For three typical cases, we also find, six CMF strengths are all positive in Fig.2(a); all negative in Fig.2(b) and three strengths are negative and three are positive in Fig.2(c). such behavior will result in co-field or anti-field rotation of the particle for ER spectra. We remark that as both the intrinsic dispersion and local-field effects are considered simutaneously, we predict four characteristic frequecies, characterized by four non-zero dispersion strengths even for $L_{z}=1 / 3$. 


\section{NUMERICAL RESULTS}

We are now in a position to calculate the dielectric dispersion, and DEP and ER spectra based on the model put forward in the previous section.

In Fig.3-5, we investigate the effect of depolarization factor $L_{z}$ on the dielectric response [denoted by $\epsilon_{e} / \epsilon_{0}$ and $\sigma_{e}$ defined in Eq.(11)], DEP and ER spectra using Eq.(22) and Eq.(23).

The dielectric response mainly exhibits two sub-dispersions characterized by two-step rapid decrease in Fig.3(a), Fig.4(a) and Fig.5(a). It is evident that the influence of $L_{z}$ (the shape) on the effective dielectric constant (or the effective conductivitty) is significant in the low-frequency region where $f<10^{4} \mathrm{~Hz}$ (or in the high-frequency region where $f>$ $10^{8} \mathrm{~Hz}$ ). This is in agreement with previous experimental conclusions that the low-frequency subdispersion for $\epsilon_{e}$ depends on the cell shape, whereas the high-frequency is independent of it [8]. The dielectric permittivity for oblate spheroidal particles, say $L_{z}=0.95$, in the low-frequency region can be as four times as the one for spherical inclusions. Thus, by taking into account the shape of cell suspensions, it is possible to reduce some discrepancies between the theoretical and experimental results on the dielectric dispersion spectra $[8,19]$.

For DEP force, it exhibits strong sensitivity to the depolarization factor (the shape). When DEP force is positive (the particles will be attracted towards the field-generating electrodes), with increasing $L_{z}$, such an attractive force becomes weak. However, when the DEP force is negative (the particles will be repelled from the electrodes), the larger $L_{z}$, the stronger repulsion will be. Furthermore, we predict one or two shape-dependent crossover frequencies at which there is no net force on the cell particle. The crossover frequency is a monotonically increasing or decreasing function of $L_{z}$, dependent on whether the variation of DEP force around it is negative or positive [see the inserts in Fig.3(b), Fig.4(b) and Fig.5(a)]. This is an interesting result. To the best of our knowledge, the dependence of crossover frequency on the spheroidal shape is reported here for the first time.

For ER spectra, they exhibit two ER characteristic frequencies with both positive strengths in Fig.3(b), both negative strengths in Fig.4(b), and negative and positive strengths in Fig.5(b) (This has been observed in experimental electrorotational spectra of the yeast cell $[26,27])$. Positive ER torque means the co-field rotation of the particle. In this case, with increasing $L_{z}$, the ER characteristic frequency for ER will be blue-shifted accompanied with the decrease of the dispersion strengths. However, when the ER torque is negative (the particle exhibits the anti-field rotation), both the ER characteristic frequencies

and the strengths decrease concomitantly with $L_{z}$. Moreover, we find that the variation of the geometric parameter $L_{z}$ does not change the polarity of ER peak.

We emphasize to point out that, although we analytically predict four and six characteristic frequencies for dielectric dispersion and ER spectra, these frequencies are mainly located in two regions or some of their dispersion strengths are quite small in comparison with others, resulting in only two effective characteristic frequencies at which the rate of change of the dielectric permittivity (or DEP force), and the rotational torque peak attain their maxima. This can be well understood from Fig.6.

As we have included the dielectric dispersion form in $\tilde{\epsilon}_{1}$, it would be interesting to investigate how the dispersion strength $\triangle \epsilon_{1}$ and the intrinsic frequency $f_{1}$ affect the dielectric dispersion and ER spectra.

In Fig.7, we examine the effect of $\triangle \epsilon_{1}$ on the dielectric response (the left panel) and 
ER spectra (the right panel). Clearly, one common feature of all these dielectric spectra is that increasing $\triangle \epsilon_{1}$ yields increasing dielectric permittivity in the low-frequency region. As previous models do negelect the intrinsic dispersion effect, the theoretical results in the low-frequency region are less than experimental reports $[8,19]$. We think it would be helpful to give the closest fit with experimental data by suitable adjustment of both $\triangle \epsilon_{1}$ and $L_{z}$. For ER spectra, when $\triangle \epsilon_{1}=0$, only one dominant peak is predicted as expected. For a finite $\triangle \epsilon_{1}$, two dominant peaks at low and high characteristic frequencies arise. With increasing $\triangle \epsilon_{1}$, the characteristic low frequency shifts towards a long wavelength; while the characteristic high frequency shifts towards a short one, leading to further separation between two characteristic frequencies for larger $\Delta \epsilon_{1}$. At the same time, the adjustment of $\triangle \epsilon_{1}$ can also change the peak value much or less and even dominate the co-field or anti-field rotation through changing the polarity of the peak (see the upper right column).

In Fig.8, we plot the dielectric dispersion and ER spectra versus the frequency $f$ for different $f_{1}$. It is evident that, $f_{1}$ mainly play a role in the characteristic high frequencies for both the effective dielectric dispersion and ER spectra. Generally speaking, the characteristic high frequency increases with increasing $f_{1}$, in accord with the analytical formula from Eq.(7). Interestingly, the increase in $f_{1}$ can also lead to the change of the polarity of the ER peak in the high-frequency region (see the middle right pannel).

In order to take into account the mutual interaction bewtween the suspended particles, we adopt Eq.(17) instead of Eq.(16). When the mutual interaction is taken into account, the ER peak must be reduced, as expected in Fig.9. With further increasing $p$, the mutual interaction becomes strong, leading to the serious depression of ER peak and the slight blueshift (or red-shift) in the characteristic low frequency (or high frequency). These results are also new.

\section{DISCUSSION AND CONCLUSION}

In this work, we have investigated both analytically and numerically the ac electrokinetic behavior, i.e., the dielectric dispersion behavior, the DEP and ER spectra of nonspherical cell suspensions with an intrinsic dispersion based on the spectral representation method. The dependence of the ac electrokinetic behavior on the depolarization factor is studied in detail. We find that an intrinsic dispersion in the dielectric constant of spheroidal cells can lead to four and six dispersion in the effective dielectric constant and the CMF respectively. We also find that both the intrinsic dispersion strength $\triangle \epsilon_{1}$ and the characteristic frequency $f_{1}$ can change the polarity of ER torque and thereby causing the co-field or anti-field rotation. Furthermore, by taking into account the local-field effect from the mutual interaction, we examine the ER spectra for various volume fractions, and find that increasing volume fractions can result in the decrease of both the strengths and the difference between two characteristic low and high frequencies indeed. Thus it is possible to obtain good agreement between theoretical predictions and experimental data by the suitable adjustment of both the geometric parameters (for example, the particle shape) and the physical parameters (for example, the dispersion strength and the characteristic frequency of cell suspensions). On the contrary, such a fitting is very useful to obtain the relevant physical information of cell suspensions.

Here a few comments are in order. We obtain four and six characteristic frequencies for 
the effective dielectric permittivity and CMF. Generally, none of the characteristic frequencies for the dielectric spectra is equal to those of CMF, as the poles $x_{1}$ and $x_{2}$ [Eq.(4)] are quite different from the poles $s_{1}, s_{2}$ and $s_{3}$ of CMF. In the dilute limit and for spherical inclusions, both Eq.(3) and Eq.(17) yield two characteristic frequencies; the smaller $p$, the less differences between these frequencies [15] are. Numerically, the dielectric dispersion, DEP and ER spectra mainly exhibit two rapid changes or two dominant peaks. Here, we also show a four-step rapid decrease in dielectric dispersion spectra (see Fig.10). However, due to small differences between the CMF frequencies or small strengths of some frequencies in comparison with others, it is difficult to show all six characteristic frequencies in ER spectra clearly. A possible way to achieve this is to consider cell suspensions with large volume fractions while being far from the spherical shape.

We demonstrate theoretically that the shape effect on the effective dielectric constant is significant in the low-frequency region, in accord with experimental conclusions [8]. In previous work, one of the authors have presented a first-principle study of the dielectric dispersion of fission yeast cell suspensions [7]. As the derivation is based on the assumption that cell suspensions are in the dilute limit and do not exhibit an intrinsic dispersion, the predicted two characteristic frequencies are independent of the volume fractions and some discrepancies between the theory and experiment on fission yeast cells still exist. We believe that our present formula can be applied also and the discrepancies between theoretical and experimental results may further be reduced.

For shelled spheroidal particles dispersed in the host medium, we have found one peak in the electrorotation assay, under the assumption that the ratio of shell to the host dielectric constant is real [11]. The theory yields good agreement with the experimental results only in the high-frequency region. In fact, for such kind of three component system, the effective dielectric response $\epsilon_{c s}$ for the coated shells can be firstly obtained in a self-consistent way [21] and will exhibit at least one dispersion. The three-component composites can then be equivalent to the solid spheroidal particles with $\epsilon_{c s}$ including one dispersion term suspended in the host medium. Thus our present model can be safely used, and the co-field rotational peak in the low-frequency rigion may also be predicted. Finally, we should remark that, for nonspherical cells, the intrinsic dispersion may depend on the orientation of a spheroid, which will yield dielectric anisotropy in cell suspensions. In this work, we take into account the geometric anisotropy as well as the dielectric isotropy. The work including both geometric and dielectric anisotropies is in progress and will be reported elsewhere.

\section{ACKNOWLEDGMENTS}

This work was supported by the RGC Earmarked Grant under project numbers CUHK 4245/01P. L. G. acknowledges the financial supports of the National Natural Science Foundation of China under Grant No.10204017 and of the Science Foundation of Jiangsu Province under Grant No. BK2002038. K.W.Y. thanks Prof. A. R. Day for his interest in our work

and his suggestion to examine the effects of inhomogeneities on the dielectric response of biological cell suspensions. 


\section{REFERENCES}

[1] For a review, see J. Gimsa and D. Wachner, Biophys. J. 77, 1316 (1999).

[2] J. Gimsa, Ann. NY Acad. Sci. 873, 287 (1999).

[3] K. Asami, T. Hanai and N. Koizumi, Jpn. J. Appl. Phys. 19, 359 (1980).

[4] G. Fuhr, J. Gimsa and R. Glaser, Stud. Biophys. 108, 149 (1985).

[5] J. Gimsa, P. Marszalek, U. Loewe and T. Y. Tsong, Biophys. J. 60, 749 (1991).

[6] K. Asami, J. Non.-Cryst. Solids 305, 268 (2002).

[7] Jun Lei, Jones T. K. Wan, K. W. Yu and Hong Sun, Phys. Rev. E 64, 012903 (2001).

[8] K. Asami, Biochim. Biophys. Acta 1472, 137 (1999).

[9] H.A.Pohl, Cambridge University Press, Cambridge, 1978.

[10] T.N.Tombs and T.B.Jones, Rev. Sci. Instrum. 62(4), April(1991).

[11] J. P. Huang and K. W. Yu, J. Phys.: Condens. Matter 14, 1213 (2002).

[12] D. J. Bergman, Phys. Rep. 43, 379 (1978).

[13] G. Schwarz, J. Phys. Chem. 66, 2636 (1962).

[14] L. Gao, Jones T.K. Wan, K.W. Yu and Z.Y. Li, J. Appl. Phys. 88, 1893 (2000).

[15] X.B. Wang, Y. Huang, R. Holzel, J.P.H. Burt and R. Pethig, J. Phys. D: Appl. Phys. 26, 312 (1993).

[16] R.D. Miller and T.B. Jones, Biophys. J. 64, 1588 (1993).

[17] R. Paul and M. Otwinowski, J. Theor. Biol. 148, 495 (1991).

[18] R. Paul and K.V.I.S. Kaler, Phys. Rev. E 48, 1491 (1993).

[19] F. Bordi, C. Cametti and T. Gili, J. Non.-Cryst. Solids, 305, 278 (2002).

[20] J. P. Huang, K. W. Yu and G. Q. Gu, Phys. Rev. E 65, 021401 (2002).

[21] L. Gao, Jones T.K. Wan, K.W. Yu and Z.Y. Li, J. Phys. Condens. Matter 12, 6825 (2000).

[22] K.R. Foster, F.A. Sauer and H.P. Schwan, Biophys. J 63, 180 (1992).

[23] C.F. Bohren and D.R. Huffman, 1983, Absorption of Scattering of Light by Small particles (New York: Wiley).

[24] J.M. Cruz and F.J. Garcia-Diego, J. Phys. D: Appl. Phys. 31, 1745 (1998).

[25] X.B. Wang, R. Pethig and T.B. Jones, J. Phys. D: Appl. Phys. 25, 905 (1992).

[26] X.F. Zhou, G.H. Markx, R. Pethig, Biochim. Biophys. Acta 1281, 60 (1996).

[27] M. Kriegmaier, M. Zimmermann, K. Wolf, U. Zimmermann and V.L. Sukhorukov, Biochim. Biophys. Acta 1568, 135 (2001). 


\section{FIGURES}

FIG. 1. The characteristic frequencies and the dispersion strengths against the depolarization factor $L_{z}$ for $p=0.2$ and (a): $s=1.625, t=-0.053, \epsilon_{2}=78 \epsilon_{0}, \sigma_{2}=10^{-5} \mathrm{~S} / \mathrm{m}, \triangle \epsilon_{1}=200 \epsilon_{0}$ and $f_{1}=1.59 \times 10^{7} \mathrm{~Hz} ;(\mathrm{b}): s=-0.031, t=1.25, \epsilon_{2}=6 \epsilon_{0}, \sigma_{2}=0.02 \mathrm{~S} / \mathrm{m}, \triangle \epsilon_{1}=1000 \epsilon_{0}$ and $f_{1}=2.65 \times 10^{5} \mathrm{~Hz} ;(\mathrm{c}): s=2.78, t=1.053, \epsilon_{2}=78 \epsilon_{0}, \sigma_{2}=2 \times 10^{-3} \mathrm{~S} / \mathrm{m}, \triangle \epsilon_{1}=2500 \epsilon_{0}$ and $f_{1}=1.9 \times 10^{6} \mathrm{~Hz}$.

FIG. 2. The characteristic frequencies $f_{b 1}=f_{c 1}\left(s_{1}\right), f_{b 2}=f_{c 2}\left(s_{1}\right), f_{b 3}=f_{c 1}\left(s_{2}\right), f_{b 4}=f_{c 2}\left(s_{2}\right)$, $f_{b 5}=f_{c 1}\left(s_{3}\right), f_{b 6}=f_{c 2}\left(s_{3}\right)$, and the dispersion strengths $\triangle b_{1}=V_{1} A\left(s_{1}\right), \triangle b_{2}=V_{1} B\left(s_{1}\right)$, $\triangle b_{3}=V_{2} A\left(s_{2}\right), \triangle b_{4}=V_{2} B\left(s_{2}\right), \triangle b_{5}=V_{3} A\left(s_{3}\right)$ and $\triangle b_{6}=V_{3} B\left(s_{3}\right)$ for CMF $\left(b_{z}\right)$ versus $L_{z}$. The parameters are same as those in Fig.1.

FIG. 3. Effective dielectric response $\left(\epsilon_{e} / \epsilon_{0}\right.$ and $\left.\sigma_{e}\right)$ (a) and normalized dielectrophoresis (NDEP) and electrorotation (NER) (b) versus the frequency $f$ for the parameters in Fig.1(a). In the insert of Fig.3(b), the crossover frequency $f_{\text {cross }}$ is plotted against $L_{z}$.

FIG. 4. Same as Fig.3, but for the parameters in Fig.1(b).

FIG. 5. Same as Fig.3, but for the parameters in Fig.1(c). Note that two shape-dependent crossover frequencies are predicted.

FIG. 6. Contributions of dielectric response $\epsilon_{e i} / \epsilon_{0} \equiv \triangle \epsilon_{e i} /\left[1+\left(f / f_{e i}\right)^{2}\right](i=1,4)$ to the dieletric spectra $\left(\epsilon_{e}-\epsilon_{H}\right) / \epsilon_{0}(\mathrm{a})$, and contributions of i-th strengths $N E R_{i} \equiv \triangle b_{i} f f_{b i} /\left(f_{b i}^{2}+f^{2}\right)$ to $N E R$ (b) versus $f$ for $L_{z}=0.05$. Other parameters are same as those in Fig.1(c). Note that the effective dieletric spectra and the $N E R$ spectra mainly exhibit two-step rapid decrease and two dominant peaks respectively, although four and six characteristic frequencies are analytically predicted.

FIG. 7. $\left(\epsilon_{e}-\epsilon_{H}\right) / \epsilon_{0}$ and $N E R$ versus $f$ for $L_{z}=0.95$ and various $\triangle \epsilon_{1}$. Other parameters are same as those in Fig.1.

FIG. 8. $\left(\epsilon_{e}-\epsilon_{H}\right) / \epsilon_{0}$ and $N E R$ versus $f$ for $L_{z}=0.95$ and various $f_{1}$. Other parameters are same as those in Fig.1.

FIG. 9. $N E R$ versus $f$ with Eq.(16) and Eq.(17) for $L_{z}=0.05$. Other parameters are same as those in Fig.5. Note that Eq.(16) is independent of $p$.

FIG. 10. $\left(\epsilon_{e}-\epsilon_{H}\right) / \epsilon_{0}$ and $N E R$ versus $f$ for $p=0.2, L_{z}=0.95$ and $\triangle \epsilon_{1}=1 \times 10^{4} \epsilon_{0}$. Other parameters are same as those in Fig.1(c). There are four characteristic frequencies, at which the dielectric spectra exhibit rapid decrease. 

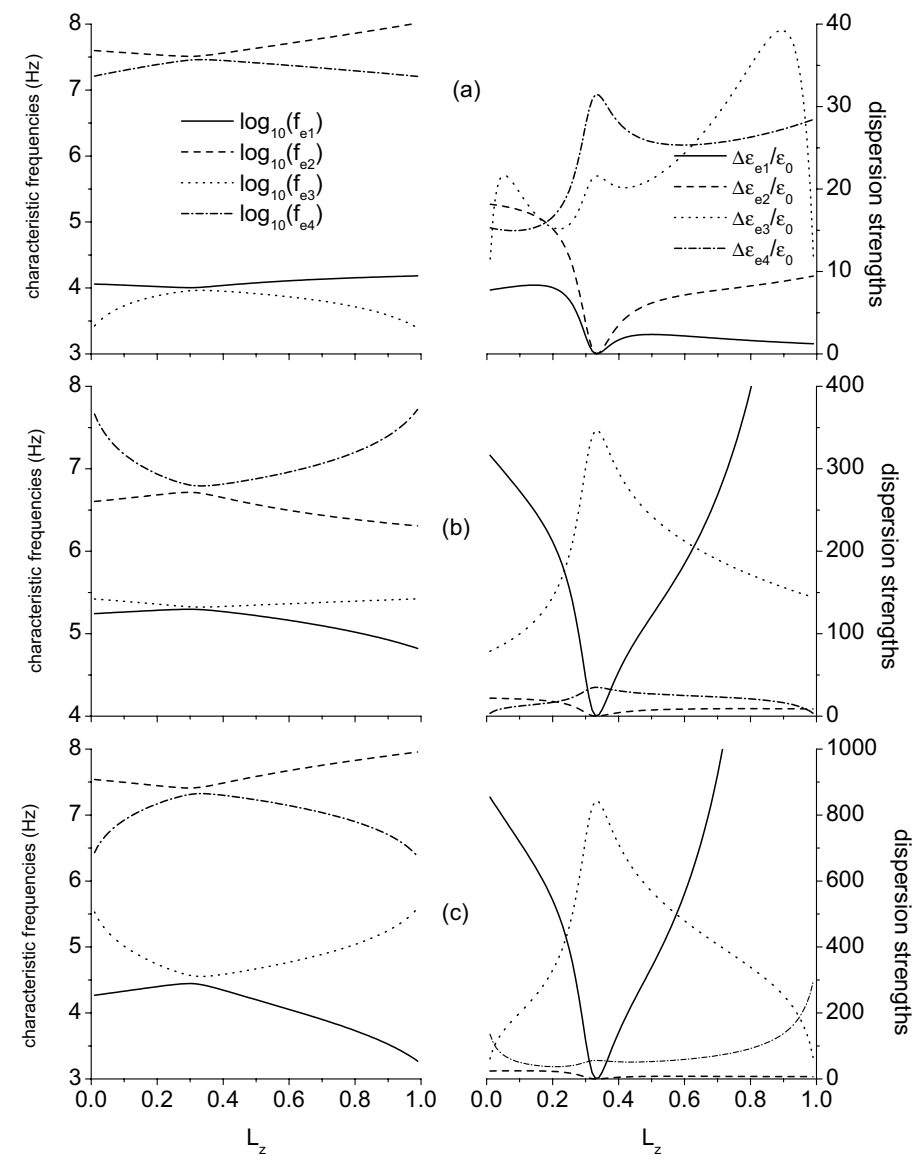

Fig.1./Gao, Huang, and Yu 

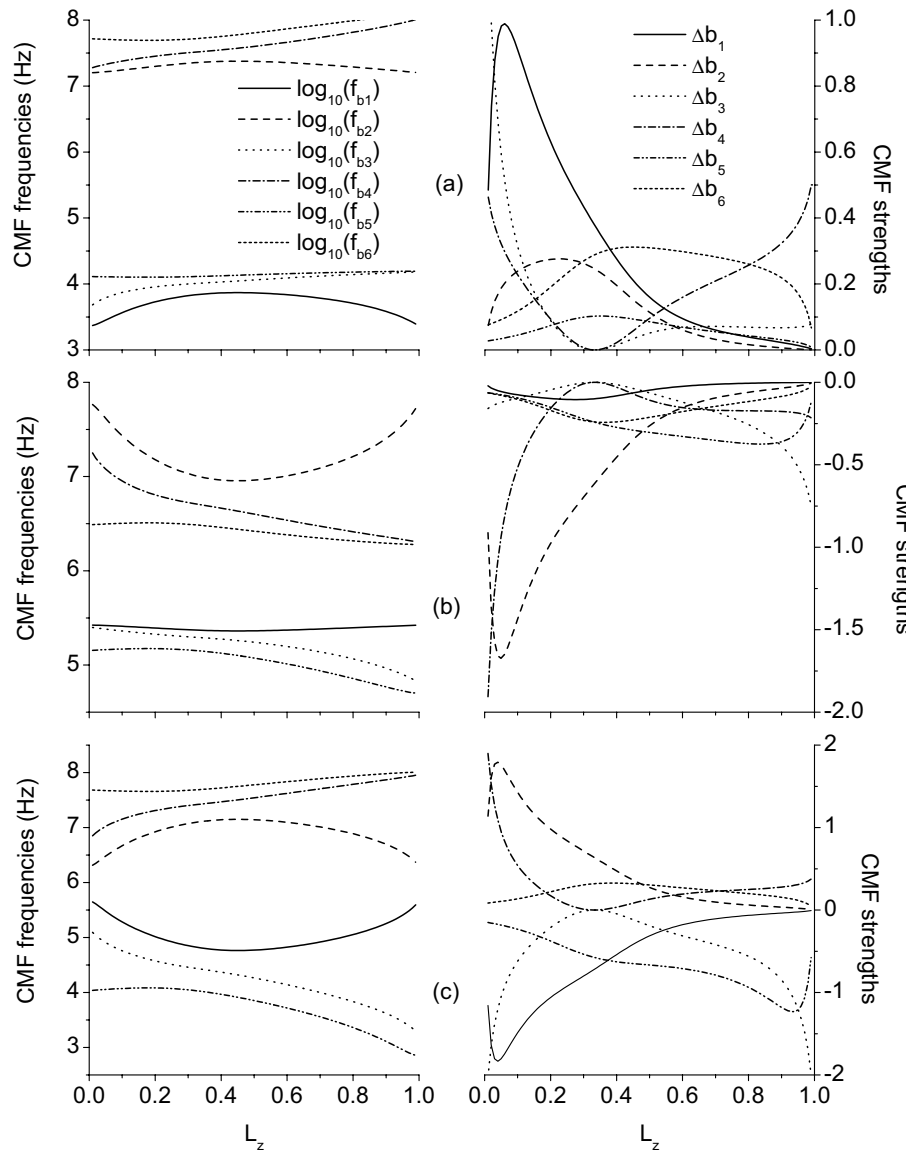

(b)
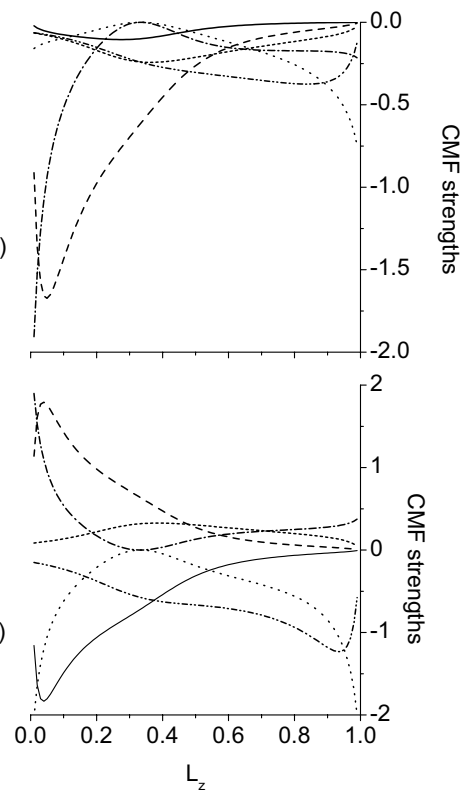

Fig.2./Gao, Huang, and Yu 


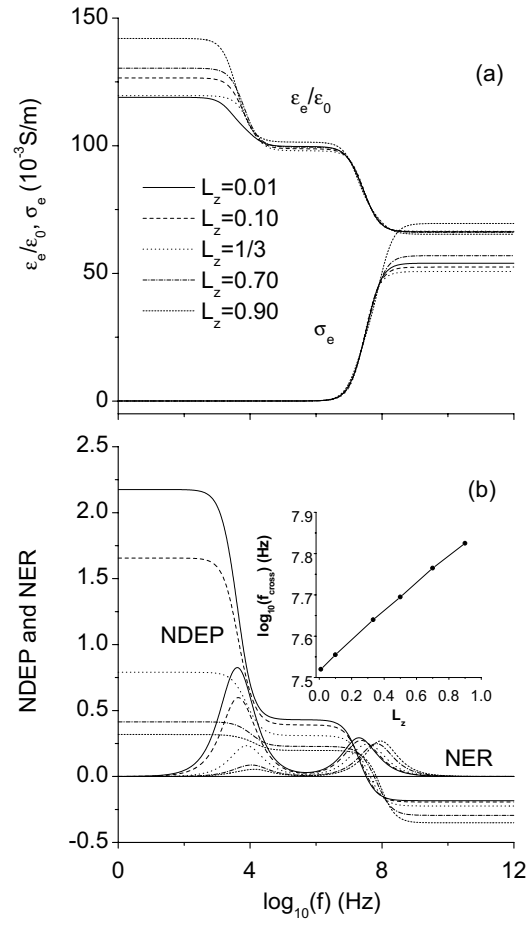

Fig.3./Gao, Huang, and Yu 

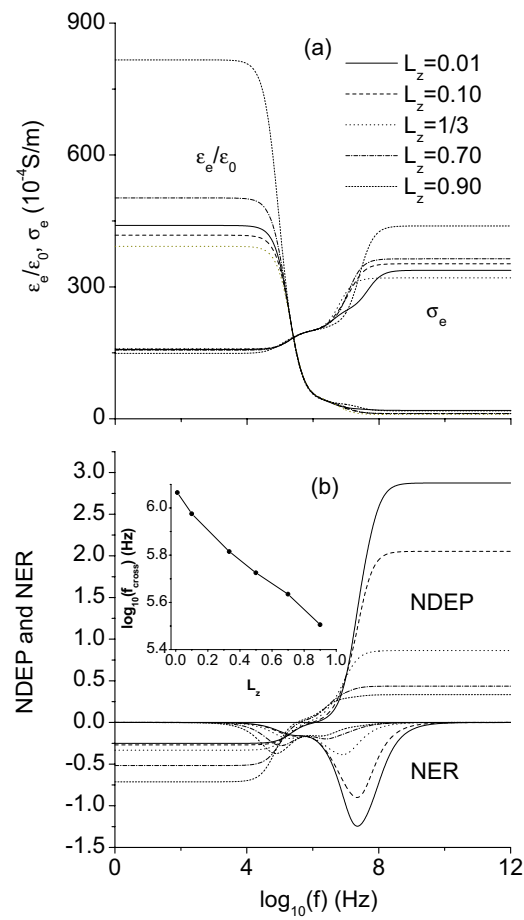

Fig.4./Gao, Huang, and Yu 


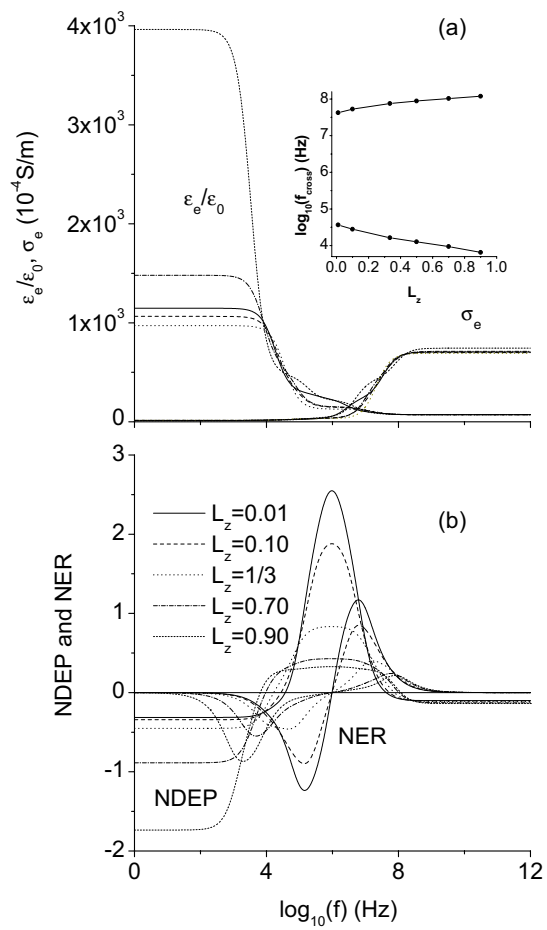

Fig.5./Gao, Huang, and Yu 

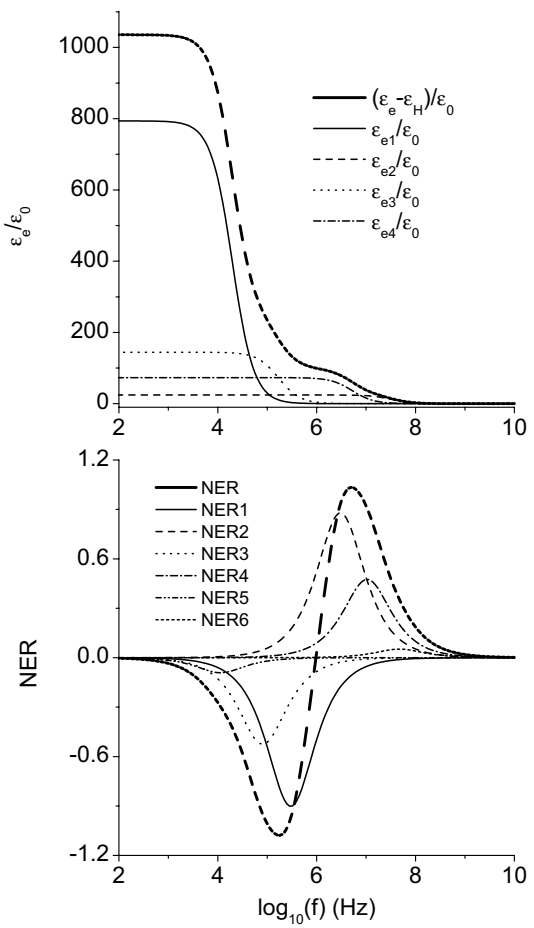

Fig.6./Gao, Huang, and Yu 

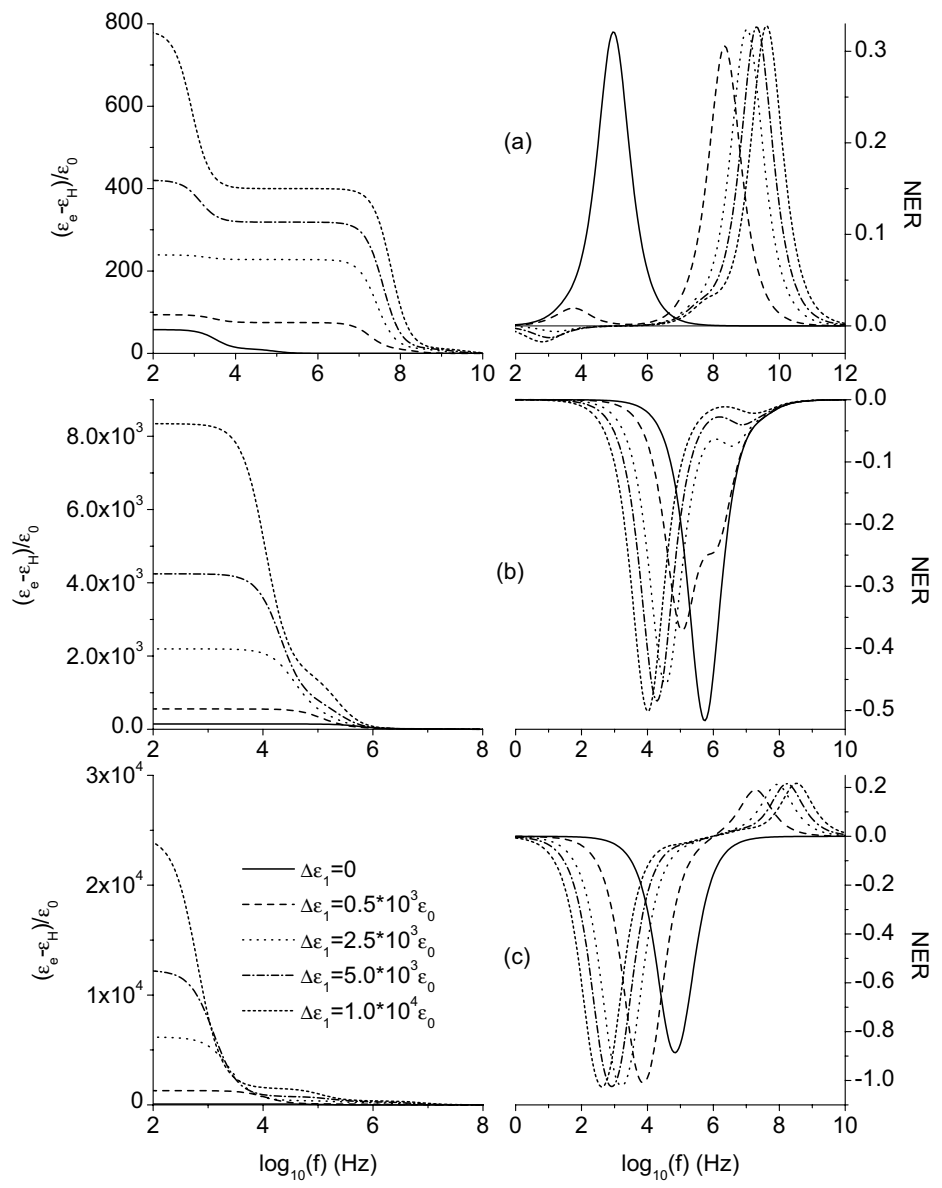

(b)
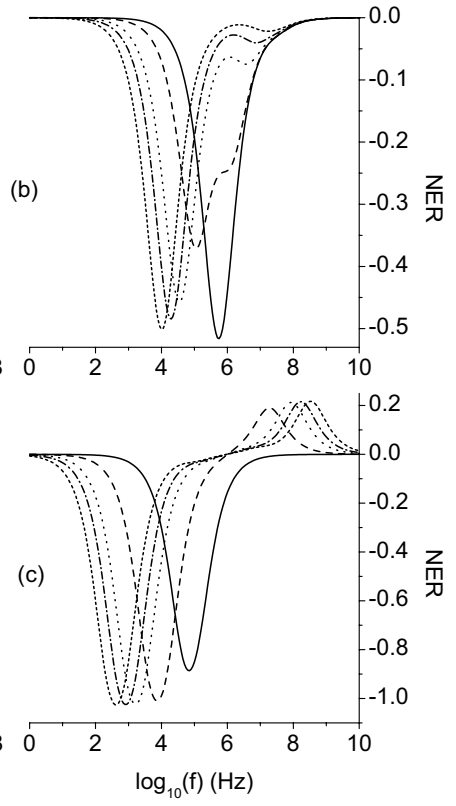

Fig.7./Gao, Huang, and Yu 

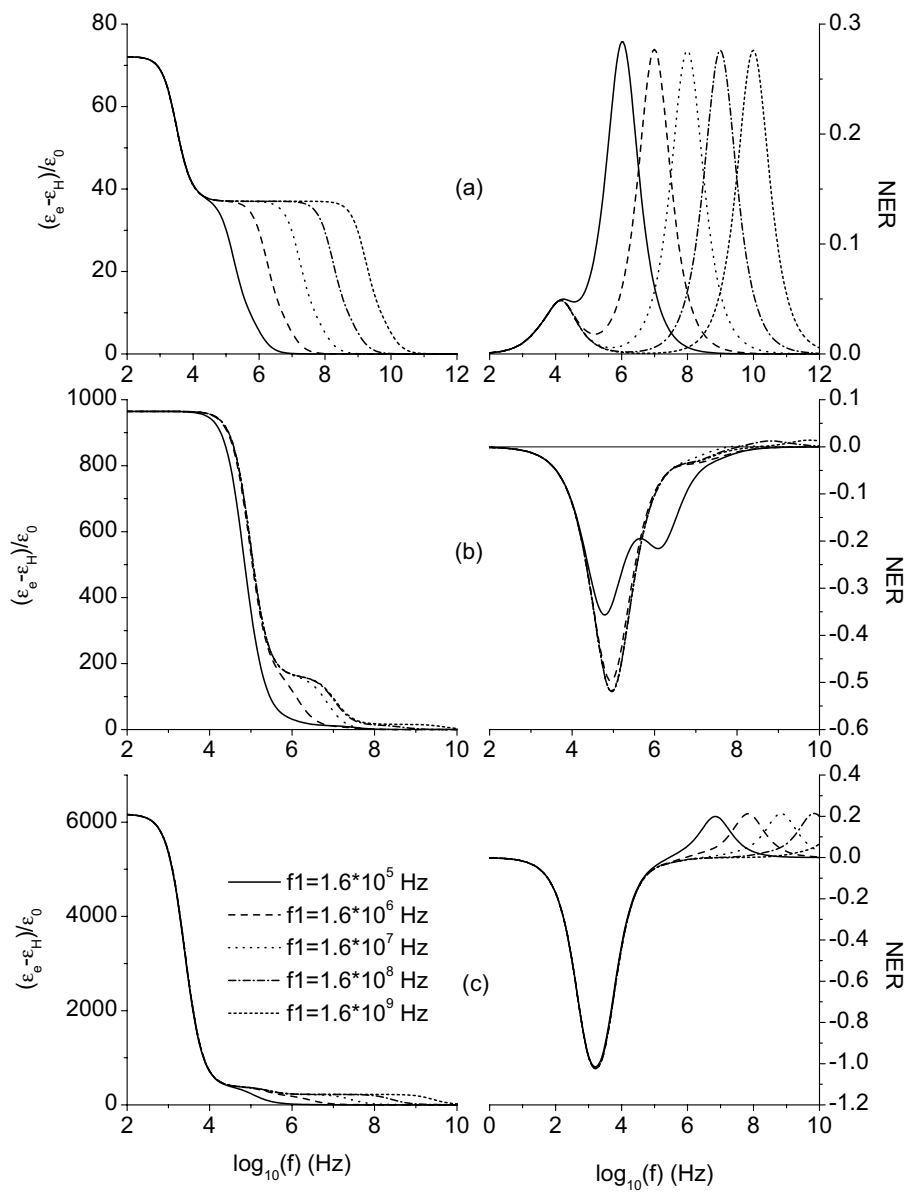

Fig.8./Gao, Huang, and Yu 


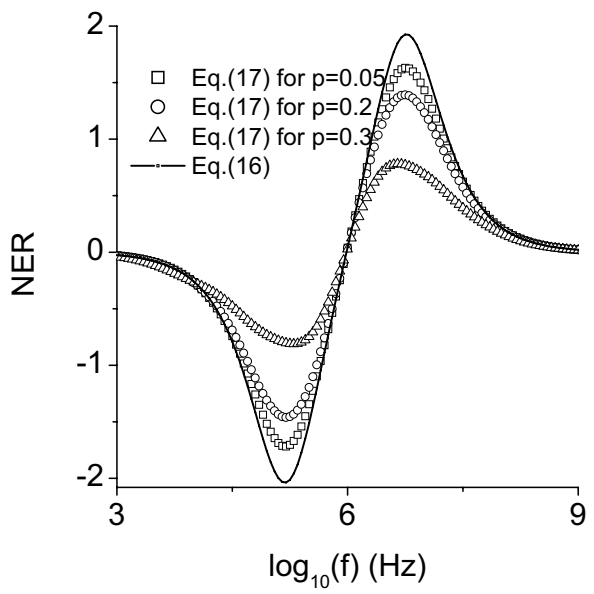

Fig.9./Gao, Huang, and Yu 


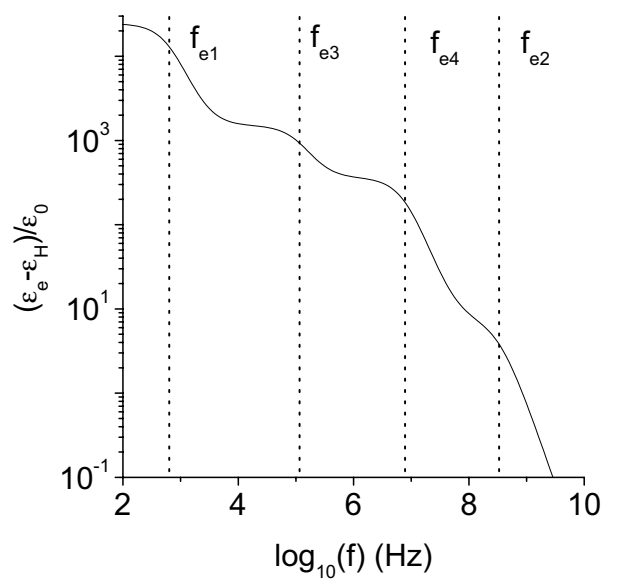

Fig.10./Gao, Huang, and Yu 